Retrospective Study

\title{
e Decompressive L5 Transverse Processectomy for Bertolotti's Syndrome: A Preliminary Study
}

Chang il Ju, MD, Seok Won Kim, MD, Jong Gyue Kim, MD, Seung Myung Lee, MD, Ho Shin, MD, and Hyeun Young Lee, MD

From: Department of Neurosurgery, Chosun University College of Medicine, Gwangju, Korea

Address Correspondence: Chang il Ju, MD Department of Neurosurgery Chosun University College of Medicine 375 Seosuk-Dong Dong-Gu Gwangju, 501-759 Korea E-mail:

jchangil@chosun.ac.kr

Disclaimer: There was no external funding in the preparation of this manuscript.

Conflict of interest: Each author certifies that he or she, or a member of his or her immediate family, has no commercial association

(i.e., consultancies,

stock ownership, equity interest, patent/licensing arrangements, etc.) that might pose a conflict of interest in connection with the submitted manuscript.

Manuscript received: 11-02-2016 Revised manuscript received: 02-22-2017

Accepted for publication: 03-02-2017

Free full manuscript: www.painphysicianjournal.
Background: Bertolotti's syndrome is a spinal disorder characterized by abnormal enlargement of the transverse process of the most caudal lumbar vertebra. The $L 5$ transverse process may be enlarged either unilaterally or bilaterally and may articulate or fuse with the sacrum or ilium. Pseudoarticulation between the transverse process of the L5 and the alar of the sacrum can cause buttock pain and leg pain. In addition, the $L 4$ exiting nerve root could be compressed by an enlarged L5 transverse process. The authors could have obtained satisfactory results from the selected cases of Bertolotti's syndrome by applying a selective transverse processectomy of the L5.

Objective: The objective of this study is to determine the effectiveness of $L 5$ transverse processectomy for symptomatic Bertolotti's syndrome.

Study Design: A retrospective study.

Methods: A total of 256 patients with Bertolotti's syndrome who had severe buttock pain and unilateral or bilateral radiating leg pain were selected. The correct diagnosis was made based on imaging studies which included computed tomography $(C T)$, plain $x$-rays, and magnetic resonance imaging (MRI). The final diagnosis was made by confirming pain relief from anesthetic block. A total of 87 patients were classified into 2 groups: group $A$ included 50 patients whose pain was relieved by block into the pseudoarticulation and group $B$ included 37 patients whose pain was relieved by block into the L4 exiting nerve root. A total of 61 cases (group A: 39 cases, group B: 22 cases) were selected as pure $\mathrm{L} 5$ transverse processectomy.

Outcome Measures: The primary outcome measures were reduction in pain scores and improvement in quality of life.

Results: Among 61 patients, there were 19 men and 42 women. The mean age of the patients was $53.2 \pm 12$ years (group A: $57.8 \pm 14$ years [ $16-86$ years], group B: $53.4 \pm 14$ years [ $27-77$ years]). The mean follow-up period was 6.5 months. The patients' mean visual analog scale (VAS) prior to surgery was $7.54 \pm 0.81$ (group A: $7.59 \pm 0.93$, group B: $7.50 \pm 0.86$ ), and the mean postoperative VAS was $2.86 \pm 1.67$ (group A: $3.82 \pm 1.59$, group B: $2.05 \pm 1.00$ ). According to Macnab's criteria, 12 patients showed excellent results (group A: 3, group B: 9), 41 patients showed good results (group A: 11, group B: 30), 6 patients showed fair results (group A: 5, group B: 1), and 2 patients showed poor results (group A: 2, group B: 0). Thus, satisfactory results were achieved in $86.89 \%$ of the cases.

Conclusion: In patients with Bertolotti's syndrome, pseudoarticulation as well as L4 nerve root compression can be the source of buttock pain and lower extremity pain. Bisectional cutting of the L5 transverse process and decompression of the $L 4$ nerve root could be an optimal treatment for Bertolotti's syndrome, and it may be easily approached by the paraspinal approach.

Limitations: This is a retrospective study and only offers one-year follow-up data for patients with Bertolotti's syndrome who have undergone L5 transverse process resection.

Key words: Bertolotti's syndrome, pseudoarticulation, L5 transverse processectomy, paraspinal approach

Pain Physician 2017; 20:E923-E932 
$D$ ertolotti's syndrome is a lumbosacral junction segmentation anomaly characterized by an abnormally enlarged transverse process of the most caudal lumbar vertebra either unilaterally or bilaterally and may articulate or fuse with the sacrum or ilium (1). The syndrome was first described by Mario Bertolotti in 1917. He was able to demonstrate the correlation of clinically-reported low back pain (LBP) with an enlarged transverse process discovered radiographically (2). The syndrome affects approximately $4-8 \%$ of the population (3). Despite its incidence, this condition is still widely debated as a cause of LBP. The differential diagnosis should always include facet and sacroiliac joint disease. Some authors have reported that there is no association between transitional vertebrae and back pain $(3,4)$. Other investigators have discovered evidence that the lumbar discs immediately above the transitional vertebrae were significantly more degenerated when compared with discs at other levels and that instability exists above the transitional vertebrae because of a weak iliolumbar ligament, eventually leading to dysfunctional motion and back pain (5-7). In some cases, a pseudoarticulation between the transverse process and the sacrum may be a source of pain. In this report, the authors describe the diagnostic evaluation and eventual minimally invasive surgical treatment of an anomalous transverse process in a patient with mechanical LBP.

\section{Methods}

\section{Patients}

We conducted a retrospective review of the case records of 87 patients with Bertolotti's syndrome who were treated with L5 transverse processectomy in our institution during the period of April 2009 to June 2011. All 87 patients were selected from a total of 256 patients with Bertolotti's syndrome who have lumbosacral transitional vertebrae (LSTV) with buttock or leg pain. The 87 patients were classified into 2 groups. Group A included 50 patients (16 men and 34 women) whose pain was relieved by an injection of local anesthetic into the pseudoarticulation (Fig. 1). Group B included 37 patients (10 men and 27 women) whose pain was relieved by a selective $L 4$ nerve root block (Fig. 2). All of the patients (both groups A and B) were treated by an injection of local anesthetic into the pseudoarticulation first. Among them were patients whose pain was not relieved enough to confirm that the pain originated from the pseudoarticulation. We retried to inject the local anesthetic on the L4 exiting nerve root as the second procedure. If the pain was relieved more effectively by the $\mathrm{L} 4$ nerve root block, we decided the additional surgical option to decompress the L4 nerve root as well as the L5 transverse processectomy (group B).

We first reviewed the preoperative lumbar spine radiographs, lumbar spine computed tomography (CT), and magnetic resonance imaging (MRI), examining whether any transitional lumbosacral vertebra was present.

Among the 87 patients, 26 cases of Bertolotti's syndrome combined with another spinal disease such as disc herniation, spinal stenosis, or spondylolisthesis that may cause buttock pain or leg pain were excluded in order to simplify the analysis. Sixty-one patients with Bertolotti's syndrome underwent pure L5 transverse processectomy and their surgical outcomes were studied. Group $A$ included 39 patients (12 men and 27 women) and group $B$ included 22 patients ( 7 men and 15 women).

We determined the existence of transitional vertebrae according to Castellvi's criteria (8). LSTV are classified into 4 types as normal, type I: dysplastic transverse process with height larger than $19 \mathrm{~mm}$, type II: incomplete lumbarisation/sacralisation, type III: complete lumbarisation/sacralisation with complete fusion with the neighboring sacral basis, type IV mixed (Fig. 3). In addition, according to the cross sectional width of the diarthroidal joint between the broad L5 transverse process and the alar of sacrum in the 3D-CT images, we classified Castellvi type II into 2 types as short joint type (sectional width of the pseudoarticulation $<1 \mathrm{~cm}$ ) and wide joint type (sectional width of the pseudoarticulation $>1 \mathrm{~cm}$ ). Among the Castellvi type I or II of transitional LSTV, the L5 transverse processectomy has been indicated in selected patients whose severe buttock pain and leg pain had been relieved significantly by local anesthetic injection (only $1 \mathrm{~mL}$ of $2 \%$ lidocaine$\mathrm{HCl}$ without steroid). It is a very important procedure for us to discriminate the pain of pseudoarticulation from other pain mechanisms. Therefore, we injected only a small amount of lidocaine- $\mathrm{HCl}(1 \mathrm{~mL})$ into the pseudoarticulation exactly and confirmed the immediate pain relief transiently, without a long-term masking effect by steroid. In addition, this injection should be targeted to the pseudoarticulation exactly not the L5 exiting nerve root beneath the enlarged transverse process. The patients whose pain had not been relieved by block to the pseudoarticulation were regarded as having pain from a different mechanism regardless of the pseudoarticulation. 


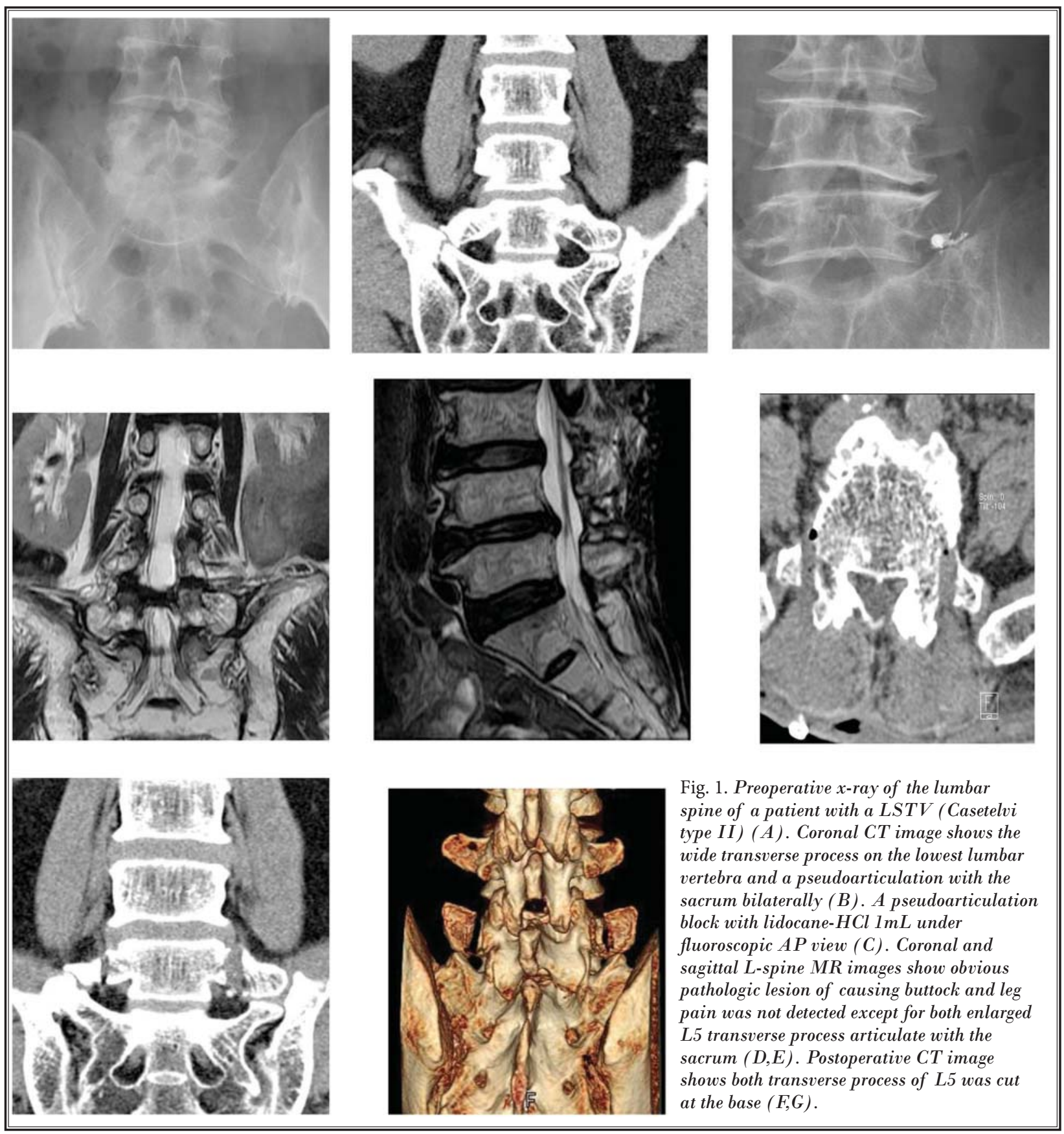

The characteristic pain from the pseudoarticulation of Bertolloti's syndrome was distributed mainly to the buttock and commonly the posterolateral thigh aspect unilaterally or bilaterally, however, lower dorsal back pain or leg pain under the knee was uncommon.

\section{Surgical Procedure}

All of the patients were brought into the operating room, where they underwent generalized endotracheal anesthesia. They were placed in the prone position on a Jackson table (OSI, Union City, CA) and the L5 spinous process was identified under fluoroscopy. An approximately $2 \mathrm{~cm}$ skin incision was made on the $3.5 \mathrm{~cm}$ lateral from the midline. Through the anatomic paraspinal intermuscular approach between the multifidus and longismus muscles, the basal part of the L5 transverse 


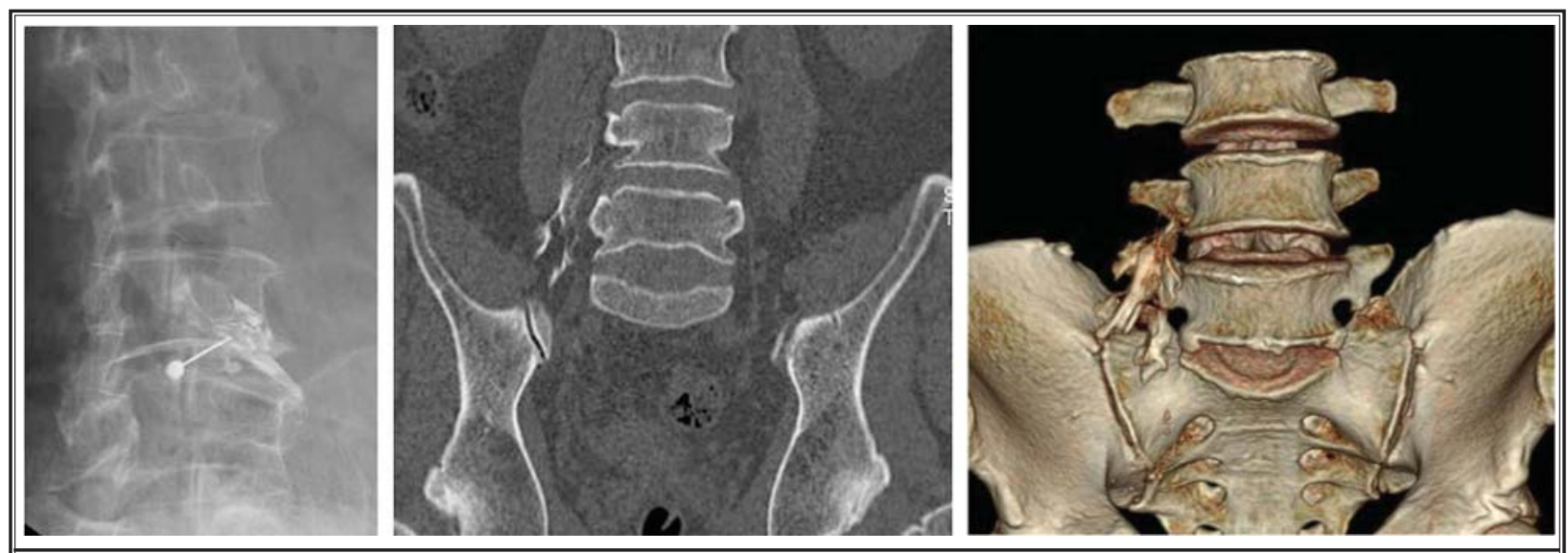

Fig. 2. Preoperative L4 nerve root block. Fluoroscopic oblique view shows the L4 nerve root after dye injection (A) and the right L4 nerve root ran crossing the front of the base part of the L5 transverse process on the CT images (B,C).

process and the upper part of the ala of sacrum were exposed. An operating microscope was used during the cutting of the L5 transverse process. In order not to cause injury to the iliolumbar ligament and the pseudoarticulation, we approached and secured the space of only the base part of the $L 5$ transverse process without exposing the lateral tip of the transverse process. We then cleared the base part of the transverse process of its muscular and ligamentous attachments. The upper and lower margins of the $L 5$ transverse process were identified by direct touching with freer. A high-speed drill was used to cut the base of the transverse process of $L 5$ bisectionally and a cutting gap of at least $0.5 \mathrm{~cm}$ was made in order not to reunite. The mechanical stress on the pseudoarticulation between the tip of the transverse process and the alar of sacrum can be blocked permanently using this procedure. The base part of the transverse process cut was coated with bone wax in order to control bone bleeding and to avoid reunion. We confirmed complete cutting by pressing the transverse process and moving it freely. In group $B$, the $L 5$ transverse processectomy was more advanced. After drilling on the base of the transverse process to cut, soft tissue was exposed and currettage to remove the soft tissue surrounding the $L 4$ exiting nerve root decompressed. We confirmed a L4 nerve root freely moving (Fig. 4).

\section{Postoperative Course}

The patients lost only approximately $25 \mathrm{~mL}$ of blood. Almost all of the patients were discharged within the following 10 days without any activity restriction. The complete cut of the base of the L5 transverse process was confirmed by postoperative CT scan.

\section{Evaluation}

Based on 3D-CT images taken prior to surgery, the size of the L5 transverse process, type of pseudoarticulation, and adjacent anatomical structures were analyzed. Approximately $1-4$ days after surgery, the area of the L5 transverse process cut was assessed by evaluation of 3D-CT images. We evaluated the surgical outcomes 3 days, one week, and 4 weeks postoperatively according to VAS score and Macnab's criteria with neurological test.

\section{Statistical Analysis}

All data were assumed to be normally distributed. We used the paired t-test to compare the difference between the preoperative and postoperative VAS scores. To compare the difference of surgical approaches between the pseudoarticulation and the L4 nerve root decompression, we conducted an analysis of 2-sample t-test. Statistical significance was defined as $P<0.05$ for all analyses. The values are given here as mean \pm standard deviation and minimum to maximum. Statistical analysis was carried out using SPSS version 21.0 (IBM Corporation, Armonk, NY).

\section{Results}

Among 256 patients with LSTV, only 87 patients (34\%) had indication for L5 transverse processotomy. The final diagnoses of these 87 cases were made by confirming immediate pain relief from anesthetic injection ( $1 \mathrm{~mL}$ of $2 \%$ lidocaine- $\mathrm{HCl}$ ) into the pseudoarticulation or the $L 4$ exiting nerve root.

Among 87 patients, a total of 61 cases (group A: 39 cases, group B: 22 cases) were selected as pure L5 trans- 


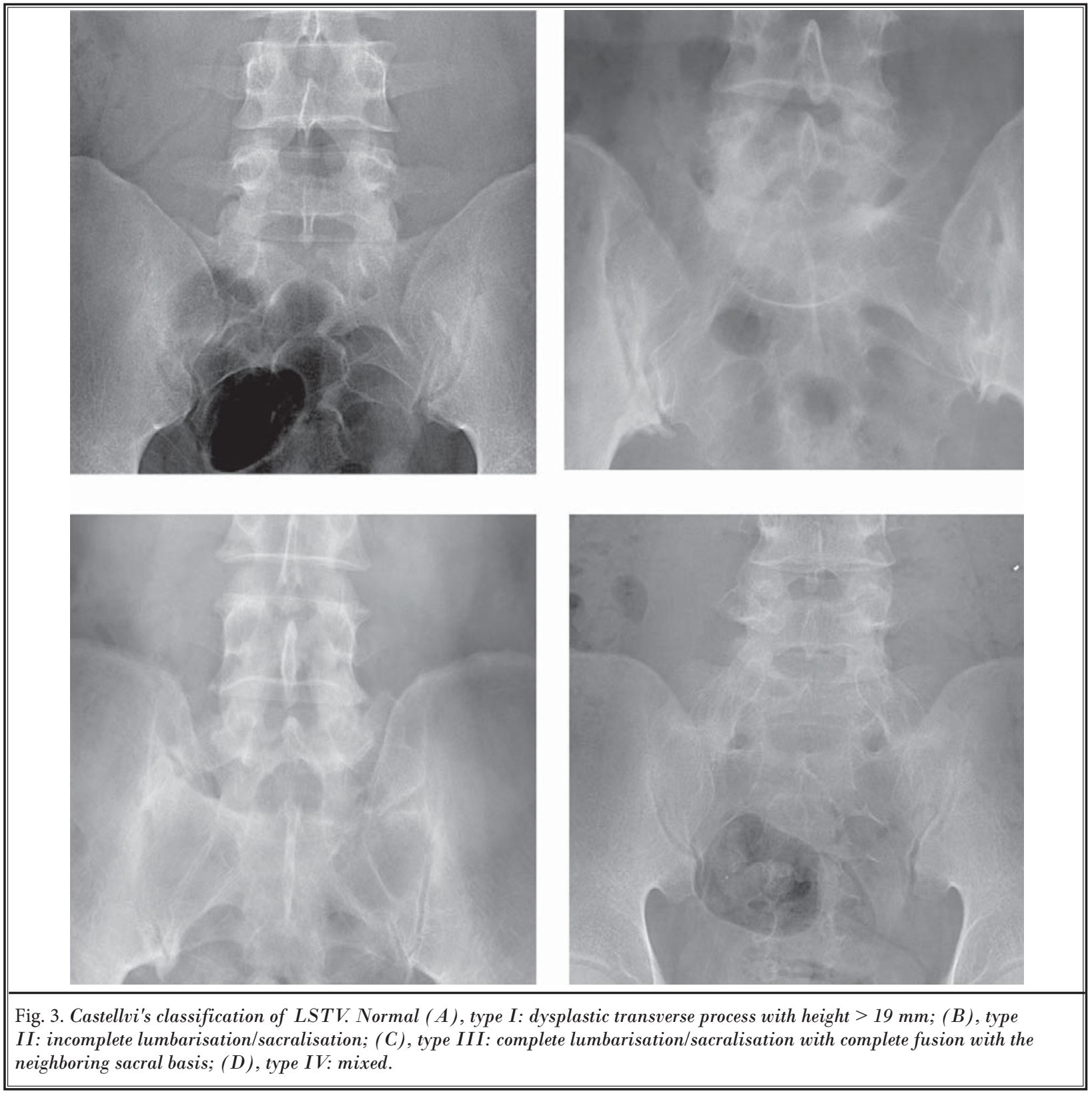

verse processectomy. The 26 patients with Bertolotti's syndrome combined with another spinal disease such as disc herniation, spinal stenosis, or spondylolisthesis that may cause buttock pain or leg pain were excluded in order to simplify the analysis. The male versus female ratio was 19:32 (group A: 12:27, group B: 7:15), and the mean age of the patients was $55.9 \pm 13.0$ years (group A: $57.14 \pm 13.3$ years, group B $54.3 \pm 12.4$ years). The average follow-up period was 10 months (range $4-30$ months).
In the 61 cases of pure L5 transverse processectomy, one patient was a teenager (group A: one, group B: 0), 2 patients were in their 20s (group A: 0, group $B: 2$ ), 4 patients were in their 30s (group $A: 3$, group $B$ : one), 13 patients were in their 40s (group A: 6, group B: 6), 13 patients were in their 50s (group A: 10, group B: 3), 11 patients were in their 60s (group A: 7, group B: 4), 12 patients were in their 70s (group $A: 8$, group $B: 2$ ), and one patient was in their 80 s (group A: one, group B: 0 ). Regarding the aspect of pain, pain occurred main- 


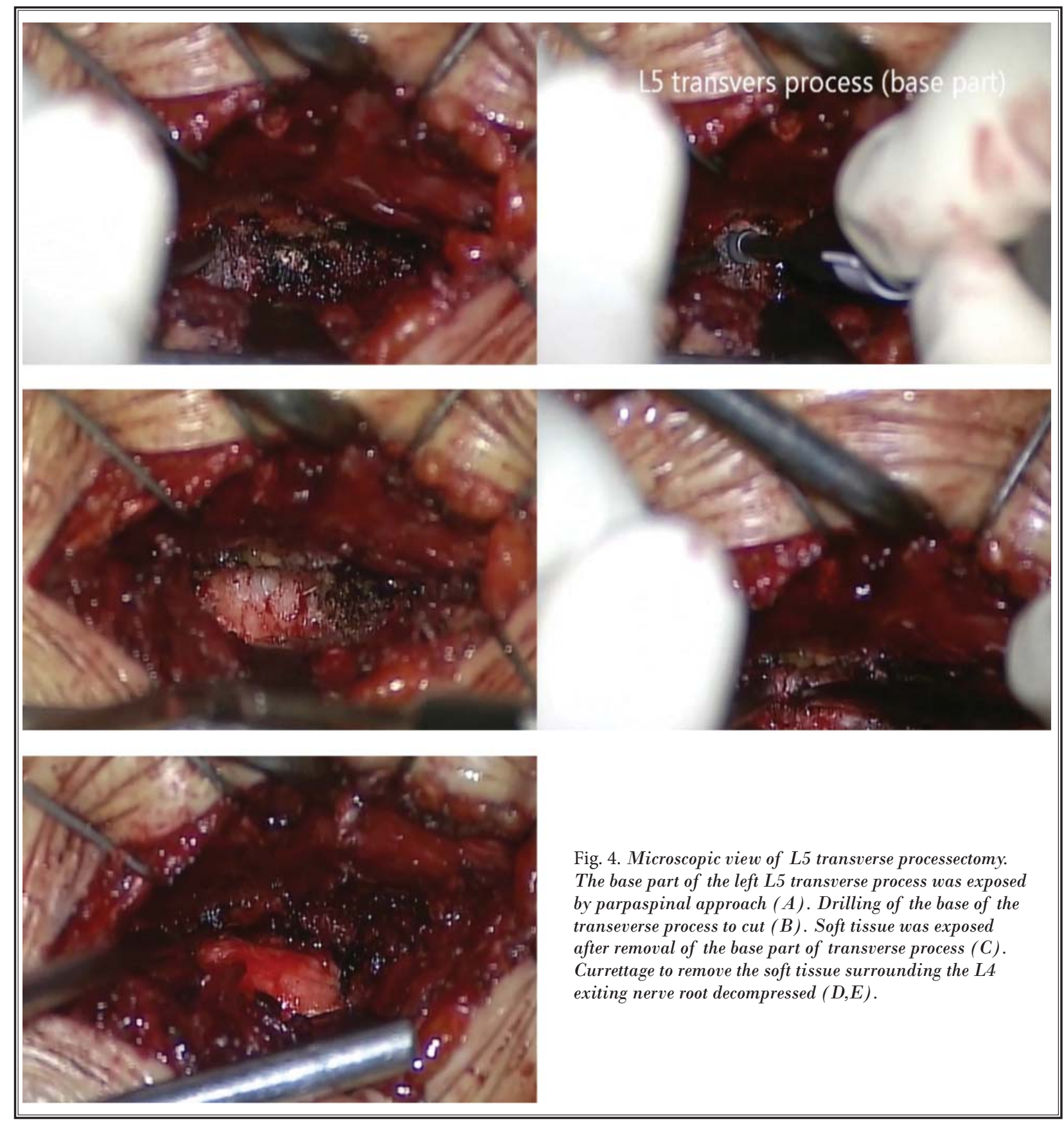

ly in the buttock (57 cases), followed by the posterior thigh (32 cases). However, LBP (19 cases) was not always observed. Rarely, buttock pain spread to the calf area (3 cases). The buttock pain was usually relieved by flexion but aggravated by extension and rotation in most of the selected patients. In addition, other symptoms would be relieved by flexion in the same manner.

According to radiographic findings, in group $A$,
11 cases were classified as Castellvi type I, and 28 cases were classified as Catellvis's type II. L5 transverse processectomy was performed bilaterally in 32 cases and unilaterally in 7 cases ( 3 on the left side and 4 on the right side).

In group A, the preoperative average VAS score was $7.59 \pm 0.93$ and the postoperative average VAS score was $3.82 \pm 1.59$ (Table 1$)$. 
However, in group $B$, the Castellvi type was not as a significant factor of pseudoarticular pain (group A). Nine cases of Castellvi type I, 7 cases of Castellvi type II, 3 cases of Castellvi type III, and also 3 cases of normal lumbosacral junction showed pain relief from the $\mathrm{L} 4$ nerve root block. In cases of L4 nerve root block, the presurgical average VAS score was $7.50 \pm 0.86$ and the postsurgical average VAS score was $2.05 \pm 1.00$. L5 transverse processectomy was performed bilaterally in 8 cases and unilaterally in 14 cases (10 on the left side and 4 on the right side).

\section{Discussion}

LSTV are common congenital anomalies of the lumbosacral spine and complete transition results in numerous abnormalities of the lumbar and sacral segments (9). LSTV have the characteristics of 2 types of abnormal vertebrae as follows. In the frequent case of sacralization of L5, the fifth lumbar vertebra fuses with the first sacral vertebra, and the person appears to have 4 lumbar vertebrae. In another case of lumbarization of S1, the first sacral vertebra may not fuse with the second, and the person appears to have 6 lumbar vertebrae $(9,10)$.

However, since it was first described by Bertolotti in 1917, the relationship between LBP with or without leg pain and the presence of LSTV has been controversial $(2,3,8,10-19)$.

In 1984, Castellvi et al (8) proposed a classification for the degree of transition based on the form and orientation of the transverse processes. According to Castellvi's classification, there are 4 types of LSTV: type I, dysplastic transverse process with height $>19 \mathrm{~mm}$, type II, incomplete lumbarization/sacralization, type III, complete lumbarization/sacralization with complete fusion with the neighboring sacral basis and type IV, mixed. The prevalence of LSTV reported in the literature ranges from $4 \%$ to more than $35 \%(20-24)$.

This wide range may be explained by the differences in diagnostic criteria, imaging techniques, and confounding factors between the investigated population samples.

In a population mainly consisting of Chinese patients using anteroposterior (AP) plain radiographs for diagnosis, Hsieh et al (16) reported a prevalence of $4 \%$. However, they excluded Castellvi type I, because this lesion would lack effects on spinal biomechanics. Erken et al (25) also used AP plain radiographs for diagnosis, but did not exclude the subtypes of LSTV. They reported a prevalence of $35.9 \%$ in a predominantly Turkish population sample. No further studies regarding racial differences have been published. In a systematic review of comparable observational studies from 1986 to 2007, Bron et al (26) reported that the mean prevalence of LSTV was $12.3 \%$. They reported that about $50 \%$ of these studies further divided LSTV in lumbarization and sacralization with a mean prevalence of $5.5 \%$ and $7.5 \%$, respectively.

Patients with LSTV are often suggested to be prone to various secondary pathologic spinal conditions including intervertebral disc herniation and/or degeneration, facet joint arthrosis, and spinal canal or foraminal stenosis $(16,23,27-32)$.

After studying 2,000 adult patients, Elster (3) found no difference in the overall incidence of structural pathology of the spine (e.g., spinal stenosis and disc protrusion) in patients with LSTV. However, Elster (3) also reported a significant difference in the distribution of these spinal lesions: bulging disc or disc herniation, as it occurred in patients with LSTV, was 9 times more common at the level immediately above the transitional vertebra compared to patients without LSTV. The increased risk for disc herniation or degeneration at the disc level above the LSTV was confirmed by other studies $(16,28,30,31)$.

Increased disc degeneration of the disc above a LSTV is attributed to its relative hypermobility $(10,33)$. This may be analogous to the advanced degeneration adjacent to a block vertebra or an interbody fusion mass $(10,23)$.

Table 1. Cases of L5 transverse processectomy of Bertolotti's syndrome.

\begin{tabular}{|c|c|c|c|c|c|c|c|c|c|c|c|c|c|c|c|c|c|c|c|}
\hline \multirow{2}{*}{$\begin{array}{c}\text { Castelvi's } \\
\text { Type }\end{array}$} & \multicolumn{2}{|c|}{ Gender } & \multicolumn{6}{|c|}{ Age Distributions } & \multicolumn{3}{|c|}{ Location } & \multirow[t]{2}{*}{ LBP } & \multirow{2}{*}{$\begin{array}{c}\text { Buttock } \\
\text { Pain }\end{array}$} & \multirow{2}{*}{$\begin{array}{c}\text { Pre- } \\
\text { op } \\
\text { VAS }\end{array}$} & \multirow{2}{*}{$\begin{array}{c}\text { Post- } \\
\text { op } \\
\text { VAS }\end{array}$} & \multicolumn{4}{|c|}{$\begin{array}{l}\text { Macnab's } \\
\text { Criteria }\end{array}$} \\
\hline & M & $\mathbf{F}$ & 10's & 20 's & 30's & 40's & 50's & 60 's & $\overline{\mathbf{B i}}$ & $\mathbf{R}$ & $\mathbf{L}$ & & & & & $\mathbf{E}$ & G & $\mathbf{F}$ & $\mathbf{P}$ \\
\hline I & 10 & 12 & 1 & 2 & 4 & 6 & 6 & 4 & 14 & 5 & 4 & 8 & 22 & 7.57 & 2.0 & 7 & 11 & 1 & \\
\hline II (short) & 9 & 15 & & 2 & 4 & 6 & 9 & 6 & 15 & 6 & 6 & 7 & 27 & 7.48 & 2.15 & 9 & 20 & 1 & 1 \\
\hline II (wide) & 9 & 12 & & & 4 & 4 & 5 & 2 & 10 & 2 & 3 & 4 & 8 & 7.4 & 5.4 & 2 & 7 & 4 & 2 \\
\hline Total & 28 & 37 & 1 & 4 & 12 & 16 & 20 & 12 & 39 & 12 & 13 & 19 & 57 & 7.5 & 2.87 & 18 & 37 & 7 & 3 \\
\hline
\end{tabular}


Konin et al (34) reported that the LBP of Bertolotti's syndrome is currently thought to be of varying etiologies from different locations: 1) disc, spinal canal, and posterior element pathology at the level above a transition $(3,16,10,23,30,35,36), 2)$ degeneration of the anomalous articulation between a LSTV and the sacrum, 3) facet joint arthrosis contralateral to a unilateral fused or articulating LSTV $(1,29)$, and 4) extraforaminal stenosis secondary to the presence of a broadened transverse process $(1,5,23,37,30)$.

In this study, all selected cases of pain etiology have been strictly limited to the degeneration of the anomalous articulation, and they were confirmed by the pain relief from an anesthetic block to the pseudoarticulation with exclusion of other pain mechanisms.

Local anesthetic infiltration of the anomalous articulation or facet joint can be used as a diagnostic tool to define the origin of pain in a patient with LSTV $(1,38,39)$.

In addition, MRI and CT are important to the diagnosis of the symptomatic Bertolotti's syndrome with pain etiologies from a pseudoarticulation. In particular, coronal MR images and reconstructed CT images are helpful to classify and measure the size and shape of a pseudoarticulation.

Many authors have reported that single-photon emission computed tomography (SPECT) is helpful in differentiating a painful articulation between the enlarged transverse process and the sacral ala or ilium (Castellvi type II) from symptomatic degenerative changes in the lumbar spine and pelvis $(12,39,40)$. Indeed, focal, markedly increased uptake at the lumbosacral articulation has been shown to correspond well with the location of the pain $(12,39,40)$. In patients with unilateral LSTV (Castellvi type Ila) and contralateral LBP, SPECT has proved to be less useful for the evaluation of contralateral facetogenic pain, while CT appears to be the most sensitive (39).

Since it was first described by Bertolotti in 1917, the association between LBP and LSTV has been disputed (2). Of the 22 reviewed observational studies (Table 2), 4 studies reported a positive correlation and 5 studies reported a negative correlation between LBP and LSTV. Therefore, the clinical challenge in patients with LSTV presenting with LBP is to determine whether an anatomical substrate related to LSTV is the underlying cause of the pain. Hypertrophic transverse processes (Castellvi type I) are generally considered to have no clinical significance and do not require further attention in clinical practice $(8,16)$. In patients with more severe types of LSTV, however, certain structures should receive particular attention during clinical assessment. First, the pain may have a discogenic origin, generated in the disc above the transitional vertebra (Castellvi types II, III, IV) (35). The bulging or herniated disc may cause nerve root compression resulting in LBP and sciatica (30).

However, nerve roots may also be compressed between the transverse segment of the LSTV and the sacral ala (Castellvi type II) (37). Second, the pain may be generated in the articulation between the enlarged transverse process and the sacral ala or ilium (Castellvi type II) (5). Third, contralateral LBP in patients with unilateral LSTV (Castellvi type Ila) may reflect facetogenic pain (1).

The treatment of patients with Bertolotti's syndrome has varied over the years. Treatment ranges from conservative, nonsurgical treatment, including steroid and local anesthetic infiltration into the anomalous lumbosacral articulations, to surgical resection of the accessory joint or posterolateral fusion of the transitional segment.

Jönsson et al (5) reported symptomatic relief in 9 of 11 patients who received an injection of local anesthetic into the anomalous articulations, however, all 9 patients subsequently underwent resection of the abnormal joint. It appears that the injection was extremely useful in the diagnosis of the syndrome but did not provide long-lasting relief.

Nonetheless, pain has not always been relieved by block to the pseudoarticulation in Bertolotti's syndrome. In our study, effective pain relief by block to the abnormal joint was observed in only nearly $25 \%$ of the patients. Most persistent pain of Bertolotti's syndrome could originate from other etiologies regardless of a pseudoarticulation, and the treatment method should be chosen according to the nature of the pain (such as far-out syndrome, foraminal stenosis, etc.).

Surgical intervention has also been advocated by several authors, including Santavirta et al (7), who studied 16 patients with LBP and radiographic evidence of anomalous lumbosacral articulations. Half of the patients underwent resection of the unilateral anomaly, whereas the other half underwent posterolateral fusion of the transitional segment. Of particular interest, despite the major differences in the operative procedures they underwent, there was no significant difference in the outcome between the 2 groups.

In our study, we selected strictly the patients with Bertolotti's syndrome whose pain originated from the 
pseudoarticulation between the enlarged transverse process of L5 and the sacral alar by anesthetic block into the pseudoarticulation, inducing a transient pain relief. The cutting of the base of the transverse process of L5 bisectionally has been performed in order to block mechanical stress to the pseudoarticulation permanently. Among the various pain etiologies of Bertolotti's syndrome, our cases have only been targeted to the pseudoarticulation; although all 61 cases showed significant pain relief from the preoperative block to pseudoarticulation, the surgical outcomes of the L5 transverse processectomy showed different results according to the type of diarthroidal joint. L5 transverse processectomy was significantly effective in cases of Castellvi type I as well as Castellvi type II of short joint type, however, not effective in the cases of Castellvi type II of wide joint type. The exact reason why the surgical results were different in types of anomalous joint is unknown. However, we suggest that the pain of a pseudoarticulation can arise from the different mechanisms regardless of pain relief by anesthetic injection. The pseudoarticulation itself can be the origin of the pain such as Castellvi type I and Castellvi type II of short joint type, and this kind of pain could be controlled by reducing mechanical stress to the pseudoarticulation. On the other hand, in the cases of Castellvi type II of wide joint type, the pseudoarticulation has already acted as a true articulated joint, and therefore, only the processotomy for blocking mechanical stress to the diarthroidal joint is not effective for relief of the pain originating from joint movement. In these cases, the posterolateral fusion of L5-S1 would be more effective.

\section{Conclusion}

In Bertolotti's syndrome, the pseudoarticulation between an anomalous enlarged transverse process and the sacral alar may be the source of pain from the buttock and even the lower extremity with or without LBP. In addition, these types of pain could be originated from the compression of the L4 exiting nerve root by an enlarged L5 transverse process. In the cases of patients whose pain was relieved by anesthetic block to the pseudoarticulation or selective L4 exiting nerve root, the bisectional cutting of the base of the transverse process of $L 5$ could be an effective treatment for Bertolotti's syndrome, and it can be easily approached by using the paraspinal route.

\section{Acknowledgments}

Jung Ju and Hyun Jeong Kwak are co-first authors and contributed equally to this manuscript. Figure 2 and 3 images courtesy of Dr. Woon Rak Son from Gachon University Gil Hospital.

\section{References}

1. Brault JS, Smith J, Currier BL. Partia lumbosacral transitional vertebra resection for contralateral facetogenic pain. Spine (Phila Pa 1976) 2001; 26:226-229.

2. Bertolotti M. Contribution to the knowledge of the defects as it relates to regional differences in the spine with special regards to the assimilation of $L_{5}$ and the sacrum. [in Italian]. Radiologique Medica (Torino) 1917; 4:113-144.

3. Elster AD. Bertolotti's syndrome revisited: Transitional vertebrae of the lumbar spine. Spine (Phila Pa 1976) 1989; 14:1373-1377.

4. MacLean JG, Tucker JK, Latham JB. Radiographic appearances in lumbar disc prolapse. J Bone Joint Surg Br 1990; 72:917-920.

5. Jönsson B, Strömqvist B, Egund N. Anomalous lumbosacral articulations and low-back pain. Evaluation and treatment. Spine (Phila Pa 1976) 1989; 14:831-834.
6. Magora A, Schwartz A. Relationship between the low back pain syndrome and x-ray findings. 2. Transitional vertebra (mainly sacralization). Scan J Rehabil Med 1978; 10:135-145.

7. Santavirta S, Tallroth K, Ylinen P, Suoranta H. Surgical treatment of Bertolotti's Syndrome. Follow-up of 16 patients. Arch Orthop Trauma Surg 1993; 112:82-87.

8. Castellvi AE, Goldstein LA, Chan DP. Lumbosacral transitional vertebrae and their relationship with lumbar extradural defects. Spine (Phila Pa 1976) 1984; 9:493-495.

9. Hughes RJ, Saifuddin A. Imaging of lumbosacral transitional vertebrae. Clin $\mathrm{Ra}$ diol 2004; 59:984-991.

10. Luoma K, Vehmas T, Raininko R, Luukkonen R, Riihimäki H. Lumbosacral transitional vertebra: Relation to disc degeneration and low back pain. Spine (Phila $\mathrm{Pa}$ 1976) 2004; 29:200-205.
11. Batt ME, Skattum N, Chong BK, Tanji JL. Posterior element pain in a adolescent schoolgirl. Br J Sports Med 1996; 30:356-358.

12. Connolly LP, Drubach LA, Connolly SA, Treves ST. Young athletes with low back pain: Skeletal scintigraphy of conditions other than pars interarticularis stress. Clin Nucl Med 2004; 29:689-693.

13. Dai L. Lumbosacral transitional vertebrae and low back pain. Bull Hosp Jt Dis 1999; 58:191-193.

14. Endo K, Ito K, Ichimaru K, Komagata $M$, Imakiire A. A case of severe low back pain associated with Richard disease (lumbosacral transitional vertebra). Minim Invasive Neurosurg 2004; 47:253-255.

15. Frymoyer JW, Newberg A, Pope MH, Wilder DG, Clements J, MacPherson B. Spine radiographs in patients with low-back pain. An epidemiological study in men. J Bone Joint Surg Am 1984; 
66-A:1048-1055.

16. Hsieh CY, Vanderford JD, Moreau SR, Prong T. Lumbosacral transitional segments: Classification, prevalence, and effect on disk height. J Manipulative Physiol Ther 2000; 23:483-489.

17. Jinnaka M. Anomaly of the spine. J Jap Orthop Assoc 1929; 4:1-41.

18. Moore BH. Sacralization of the fifth lumbar vertebra. J Bone Joint Surg 1925; 7:271-278.

19. Peterson CK, Bolton J, Hsu W, Wood A. A cross-sectional study comparing pain and disability levels in patients with low back pain with and without transitional lumbosacral vertebrae. J Manipulative Physiol Ther 2005; 28:570-574.

20. Hahn PY, Strobel JJ, Hahn FJ. Verification of lumbosacral segments on MR images: Identification of transitional vertebrae. Radiology 1992; 182:580-581.

21. O'Driscoll CM, Irwin A, Saifuddin A. Variations in morphology of the lumbosacral junction on sagittal MRI: Correlation with plain radiography. Skeletal Radiol 1996; 25:225-230.

22. Peh WC, Siu TH, Chan JH. Determining the lumbar vertebral segments on magnetic resonance imaging. Spine (Phila Pa 1976) 1999; 24:1852-1855.

23. Vergauwen S, Parizel PM, van Breusegem L, Van Goethem JW, Nackaerts Y, Van den Hauwe L, De Schepper AM. Distribution and incidence of degenerative spine changes in patients with a lumbo-sacral transitional vertebra. Eur Spine J 1997; 6:168-172.

24. Wigh RE, Anthony HF Jr. Transitional lumbosacral discs: Probability of herniation. Spine (Phila Pa 1976) 1981; 6:168-171.
25. Erken E, Ozer HT, Gulek B, Durgun B. The association between cervical rib and sacralization. Spine (Phila Pa 1976) 2002; 27:1659-1664.

26. Bron JL, van Royen BJ, Wuisman PI. The clinical significance of lumbosacral transitional anomalies. Acta Orthop Belg 2007; 73:687-695.

27. Aihara T, Takahashi K, Ogasawara A, Itadera $\mathrm{E}$, Ono Y, Moriya H. Intervertebral disc degeneration associated with lumbosacral transitional vertebrae: A clinical and anatomical study. J Bone Joint Surg Br 2005; 87-B:687-691.

28. Leboeuf C, Kimber D, White K. Prevalence of spondylolisthesis, transitional anomalies and low intercrestal line in a chiropractic patient population. J Manipulative Physiol Ther 1989; 12:200-204.

29. Nicholson AA, Roberts GM, Williams LA. The measured height of the lumbosacral disc in patients with and without transitional vertebrae. $\mathrm{Br}$ J Radiol 1988; 61:454-455.

30. Otani K, Konno S, Kikuchi S. Lumbosacral transitional vertebrae and nerveroot symptoms. J Bone Joint Surg $\mathrm{Br}$ 2001; 83-B:1137-1140.

31. Paajanen $H$, Erkintalo $M$, Kuusela $T$, Dahlstrom S, Kormano M. Magnetic resonance study of disc degeneration in young low-back pain patients. Spine (Phila Pa 1976) 1989; 14:982-985.

32. Tertti MO, Salminen JJ, Paajanen HE, Terho PH, Kormano MJ. Low-back pain and disk degeneration in children: A case-control MR imaging study. Radiology 1991; 180:503-507.

33. Gay RE, Ilharreborde B, Zhao K, Zhao C, An KN. Sagittal plane motion in the hu- man lumbar spine: Comparison of the in vitro quasistatic neutral zone and dynamic motion parameters. Clin Biomech (Bristol, Avon) 2006; 21:914-919.

34. Konin GP, Walz DM. Lumbosacral transitional vertebrae: Classification, imaging findings, and clinical relevance. AJNR Am J Neuroradiol 2010; 31:1778-1786.

35. Quinlan JF, Duke D, Eustace S. Bertolotti's syndrome. A cause of back pain in young people. J Bone Joint Surg (Br) 2006; 88-B:1183-1186.

36. Taskaynatan MA, Izci Y, Ozgul A, Hazneci B, Dursun H, Kalyon TA. Clinical significance of congenital lumbosacral malformations in young male population with prolonged low back pain. Spine (Phila Pa 1976) 2005; 30:E210-E213.

37. Hashimoto $\mathrm{M}$, Watanabe $\mathrm{O}$, Hirano $\mathrm{H}$. Extraforaminal stenosis in the lumbosacral spine. Efficacy of MR imaging in the coronal plane. Acta Radiol 1996; 37:610-613.

38. Marks RC, Thulbourne T. Infiltration of anomalous lumbosacral articulations. Steroid and anesthetic injections in 10 back-pain patients. Acta Orthop Scand 1991; 62:139-141.

39. Pekindil G, Sarikaya A, Pekindil Y, Gültekin A, Kokino S. Lumbosacral transitional vertebral articulation: Evaluation by planar and SPECT bone scintigraphy. Nucl Med Commun 2004; 25:29-37.

40. Pekindil G, Pekindil Y, Sarikaya A. Degenerative lumbosacral transitional articulation: Atypical increased sacral uptake on planar bone scintigraphy. Clin Nucl Med 2002; 27:840-841. 